\title{
Análise térmica por meio de simulação computacional da influência da floresta ciliar sobre uma cidade hipotética
}

\author{
Siviero, A. F. T. ${ }^{*}$; Barros, I.H.A. ${ }^{1}$; Galuppo, M. V. ${ }^{1}$; Marcellino, M.M.A. ${ }^{1}$ \\ 1 Programa de Graduação em Engenharia Química, Universidade Federal do Espírito Santo, Alegre, ES, Brasil. \\ * e-mail: anaflaviatsiviero@gmail.com
}

\begin{abstract}
Resumo
Este estudo iniciou-se com o objetivo de analisar a influência de uma floresta ciliar à uma cidade quanto à distribuição de temperatura. Para isso, estudou-se a transferência de calor por convecção entre a cidade e a floresta, além de geração de energia interna e transferência de calor por condução na cidade.

A fim de esquematizar a situação, utilizou-se o modelo matemático da equação da difusão de calor. Feitas as devidas considerações, utilizou-se o método de diferenças finitas na discretização da equação com o propósito de resolvê-la em ferramenta computacional.
\end{abstract}

Observou-se, à partir do gráfico gerado computacionalmente, que as temperaturas são mais amenas quanto mais próximas da floresta. Isso indica que, de fato, há fluxo de calor nessa direção. Portanto, conclui-se que para evitar atingir altas temperaturas, deve-se ter em mente a importância das matas ciliares e da arborização das áreas urbanas.

\begin{abstract}
This study was started aiming to analyze a riparian forest influence on a city temperature distribution. As such, the heat transfer by convection between the city and the forest, the generation of internal energy and heat conduction in the city were studied.

A mathematical model of the heat difusion equation was used, and with proper considerations, the finite differences method was used to discretize the equation so it could be solved by a computational tool.

It was observed by the graphic generated from it that temperatures are lower in areas closer to the forest. That points to the existence of a heat flux in that direction. Therefore, it is concluded that to avoid high temperatures, the importance of such forests and urban arboring should be kept in mind.
\end{abstract}

Keywords (Palavras chaves): Heat transfer, city, model.

\section{Introdução}

Devido ao aumento da industrialização e da área urbana, as cidades sofrem com a elevação da temperatura e da poluição, que prejudicam a qualidade de vida humana. Planejamentos ambientais prévios podem amenizar esses efeitos. [1]
As ilhas de calor urbanas são os principais fatores de modificação atmosférica relativa ao processo de urbanização. O aumento da temperatura do ar nas cidades, em relação ao seu ambiente vizinho, gerado por alterações das características térmicas das superfícies, decorrentes da substituição, pelo homem, de áreas verdes por edificações e pavimentação, é 
usualmente definido como o efeito da "ilha de calor urbana". [2]

As cidades que possuem florestas em suas proximidades sofrem influência na distribuição de temperatura. Isso ocorre, pois a temperatura na floresta é, normalmente, inferior à temperatura do ar na cidade. Cidades localizadas à margem de locais arborizados possuem uma temperatura mais amena. Além disso, as florestas podem contribuir na redução da poluição gerada nas cidades e mantém a umidade do ar. [1]

A radiação térmica é a única transferência de calor que não requer a presença de um meio material. A energia do campo de radiação é transferida por ondas eletromagnéticas e por definição, a radiação é a energia emitida pela matéria que se encontra a uma temperatura diferente de $0 \mathrm{~K}$. A condução é definida como a transferência de energia das partículas mais para as menos energéticas de uma substância, devido às interações entre elas. [3]

\section{Objetivo}

O objetivo deste trabalho é simular os efeitos da transferência de calor entre florestas e uma cidade, adotando considerações pertinentes, a fim de propor um modelo matemático para auxiliar no desenvolvimento.

\section{Metodologia}

A análise da distribuição da temperatura da cidade, com influência da floresta ciliar, requer a construção de um modelo representativo do contexto do problema. Para isso, considerou-se o conjunto "floresta - cidade floresta" como uma placa plana, sendo o eixo "x" e "y" a largura e altura, respectivamente, com fluxo de calor bidimensional.

Como o problema apresenta simetria no gradiente de temperatura da cidade, isto é, sofre a mesma influência da floresta em ambos os lados, analisou-se o fluxo de calor apenas em um lado da cidade, sendo uma extremidade a interface entre a floresta e a cidade e a outra, o centro da cidade. Assim, a representação gráfica em forma de malha é:

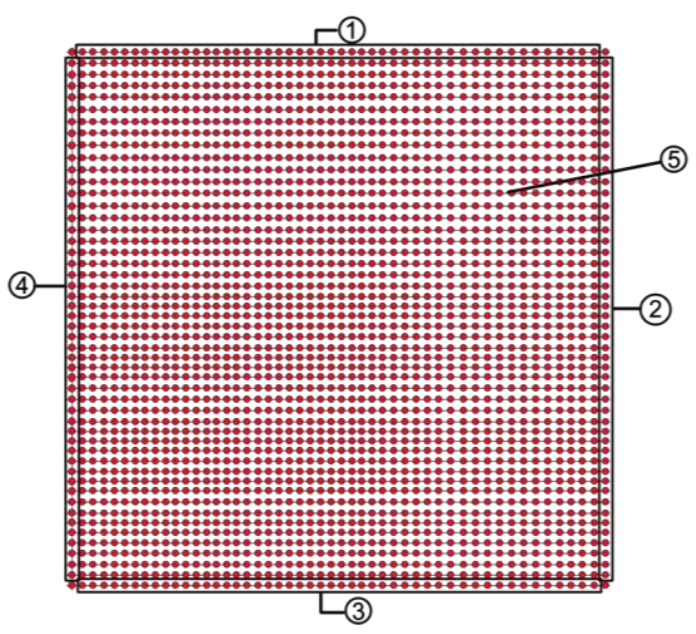

Figura 1 - Representação por malha.

Sendo:

- Grupo 1: o céu;

- Grupo 2: o centro da cidade;

- Grupo 3: o chão;

- Grupo 4: a interface entre a floresta e a cidade;

- Grupo 5: a cidade.

A relação matemática da analise da condução do calor é dada pela Equação de Calor:

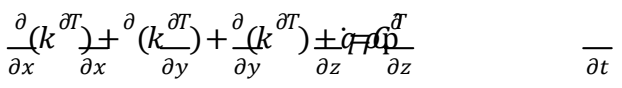

Para efeito de estudo, determinaram-se as seguintes considerações ao problema:

- Regime Transiente;

- Convecção apenas na direção x;

- Ausência de fluxo de calor na direção z;

- Temperatura da floresta: $\mathrm{T}_{\text {inf }}=20^{\circ} \mathrm{C}$;

- Dimensões da cidade: Altura $=$ Largura $=1000$ $\mathrm{km}$;

- Geração de calor uniforme na cidade: 11549.9 W (Tabela 1);

- Convecção do ar: $h=200 \mathrm{~W} /\left(\mathrm{m}^{2} \mathrm{~K}\right)$ [3]; 
- Condutividade térmica do ar: $\mathrm{k}=26.4 \mathrm{~W} / \mathrm{km} . \mathrm{K}$ [3];

- $\quad$ Capacidade calorífica do ar: 1,013 kJ/kg.K [4];

- Densidade do ar: $\rho=1.1614 \mathrm{~kg} / \mathrm{m}^{3}$ [3].

Dessa forma, a Equação de Calor se reduz a:

$$
\frac{\partial^{2} T}{\partial^{2}}+\frac{\partial^{2} T}{\partial^{2}}+{ }_{k}^{q}=\frac{Q q d}{k} \partial t
$$

Sendo:

- $\quad T=$ Temperatura;

- $\quad \mathrm{q}=$ Calor gerado na cidade;

- $\quad \mathrm{k}=$ Condutividade térmica;

- $\rho=$ Densidade do ar;

- $\mathrm{C}_{\mathrm{P}}=$ Capacidade calorífica;

- $\mathrm{t}=$ Tempo.

Como esse modelo é uma equação diferencial de segunda ordem, têm-se quatro condições de contorno e uma condição inicial. São essas:

\section{- Condições de Contorno:}

1) $T(x, n)=20^{\circ} \mathrm{C}$ (condição de Dirichlet);

2) $\mathrm{T}(\mathrm{n}, \mathrm{y})=30^{\circ} \mathrm{C}$ (condição de Dirichlet);

3) $\frac{\partial T}{\partial x}=0$ (condição de Neumann);

4) $\mathrm{K}_{-}^{\partial T}=\mathrm{h}\left(\mathrm{T}-\mathrm{T}_{\mathrm{inf}}\right)$ (condição de Robin).

- Condição inicial: $\mathrm{T}=30^{\circ} \mathrm{C}$ para $\mathrm{t}=0$.

Determinou-se o valor de geração interna de calor pela idealização de uma cidade hipotética, à partir dos seguintes dados de fontes geradoras de calor:

Tabela 1: Calor gerado em uma cidade hipotética.

\begin{tabular}{|c|c|c|c|}
\hline $\begin{array}{c}\text { Fonte de } \\
\text { calor }\end{array}$ & Quantidade & $\begin{array}{c}\text { Valor } \\
\text { Unitário } \\
\text { (W) }\end{array}$ & $\begin{array}{c}\text { Valor } \\
\text { total (W) }\end{array}$ \\
\hline Banco & 1 & 146.5 & 146.5 \\
\hline $\begin{array}{c}\text { Escola } \\
\text { primária }\end{array}$ & 1 & 102.3 & 102.3 \\
\hline
\end{tabular}

\begin{tabular}{|c|c|c|c|}
\hline $\begin{array}{c}\text { Escola } \\
\text { secundária }\end{array}$ & 1 & 116.3 & 116.3 \\
\hline Escritório & 2 & 131.4 & 262.8 \\
\hline Fabrica (leve) & 1 & 219.8 & 219.8 \\
\hline Farmácia & 2 & 146.5 & 293 \\
\hline Ginásio & 1 & 424.5 & 424.5 \\
\hline Hotel & 1 & 131.4 & 131.4 \\
\hline Loja & 1 & 131.4 & 131.4 \\
\hline Residência & 5 & 131.4 & 657 \\
\hline Restaurante & 1 & 161.7 & 161.7 \\
\hline & & TOTAL: & 2646.7 \\
\hline
\end{tabular}

Fonte: MSPC. Tabela de locais ou atividades. Disponível em: <http://www.mspc.eng.br/termo/cterm_pess_00.shtml>. Acesso em 19 de setembro de 2016.

Portanto, considera-se o a geração de calor na cidade, $\dot{q}$, igual à $11549.9 \mathrm{~W}$.

Objetivando a construção do algoritmo, discretizou-se apenas as diferenciais no espaço (" $x$ " e " $y$ ") pelo Método de Diferenças Finitas Centrais.

Para a implementação do código, definiu-se $A={ }^{q},{ }_{k}, B=$ $\frac{\rho C p}{K} \mathrm{e} d T=\frac{\partial T}{\partial t}$.

Equações discretizadas de acordo com a divisão dos grupos da malha:

- Grupo 5 (domínio): $2 \leq \mathrm{i} \leq \mathrm{n}-1 ; 2 \leq \mathrm{j} \leq \mathrm{n}-1$

$d T=\frac{\left(\frac{T(i+1, j)-2 T(i, j)+T(i-1, j)}{\Delta x^{2}}+\frac{T(i+1)-2(i)+(i(-1)}{\Delta y^{2}}+A\right)}{B}$

- $\quad$ Grupo 1: $n=1 ; 2 \leq j \leq n-1$;

$\mathrm{dT}(\mathrm{n}, \mathrm{j})=0$;

- $\quad$ Grupo 2: $2 \leq i \leq n-1 ; \mathrm{j}=\mathrm{n}$;

$\mathrm{dT}(\mathrm{i}, \mathrm{n})=0$; 


\section{SEMANA DE ENGENHARIA QUÍMICA UFES}

\section{Blucher}

- $\quad$ Grupo $3: \mathrm{i}=1 ; 2 \leq j \leq n-1$;

$$
\mathrm{dT}(1, \mathrm{j})=0
$$

- $\quad$ Grupo $4: \mathrm{j}=1 ; 2 \leq i \leq n-1$

$d T=\left(\frac{T(i+1,1)-2 T(i, 1)+T(i-1,1) T(i 2)-2 T(i)+T(\Delta))}{\Delta x^{2}}+{ }^{4} A\right.$

B (4)

Condição de contorno a ser considerada:

$$
\mathrm{k}_{d}^{d T}=\mathrm{h}(\mathrm{T}-\mathrm{T} \infty) \Rightarrow \mathrm{k} \frac{(\mathrm{T}(\mathrm{i}, \mathrm{j}+1)-\mathrm{T}(\mathrm{i}, \mathrm{j}-1))}{2 \Delta y}=\mathrm{h}(\mathrm{T}(\mathrm{i}, \mathrm{j})-
$$

$\mathrm{T} \infty)$

$$
\mathrm{T}(\mathrm{i}, 0)=\frac{-h 2 \Lambda y\left(T(i, j)-T_{\infty}\right)}{k}+\mathrm{T}(\mathrm{i}, 2)
$$

(6)

Considerando o termo $\frac{-h 2 \Delta y}{k}=\mathrm{C}$ e aplicando a condição de contorno em (4) :

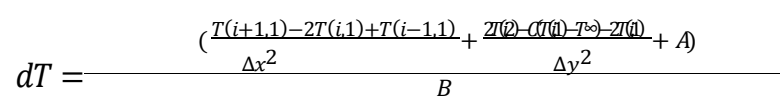

Com base nas equações e especificações definidas, criou-se um algoritmo que retorna, ao final da sua implementação, o gráfico do gradiente de temperatura da cidade.

\section{Resultados e Discussão}

Ao final da implementação do algoritmo, obteve-se o seguinte gráfico:

Figura 2: Gráfico de perfil de temperaturas da cidade.

De acordo com o gráfico, tem-se uma analogia à figura 1 e percebe-se que as temperaturas iniciais $15^{\circ} \mathrm{C}$ e $30^{\circ} \mathrm{C}$, do céu e do centro/chão da cidade, respectivamente, mantêm-se inalteradas. Porém, é possível visualizar um fluxo de calor na direção do centro da cidade para a interface entre cidade $x$ floresta, o que comprova que a cidade está perdendo calor para a floresta, que se encontra em uma temperatura inferior.

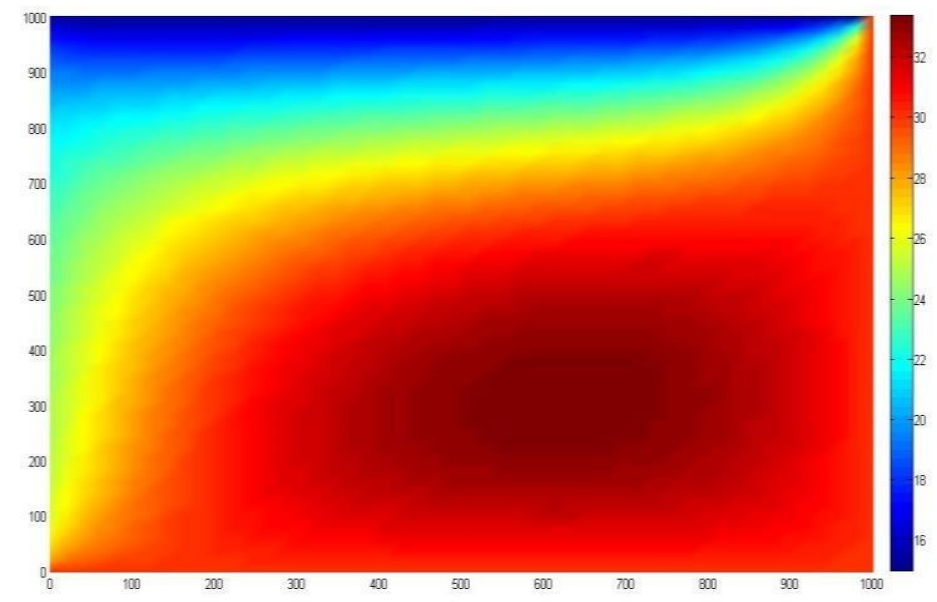

No gráfico, a região de cor vermelho escuro indica a geração de energia interna uniforme proposta nas condições pré estabelecidas da análise em questão. E a região de cor azul escuro indica o céu, que é onde a temperatura é menor em todo o gráfico.

Dessa forma, têm-se a floresta como um sumidouro de energia, isto é, um agente responsável pelo resfriamento da cidade.

De acordo com estudos de Leal (2012), realizados na cidade de Curitiba, Paraná, as regiões com maior quantidade de remanescentes florestais apresentaram menores temperaturas, além de que a distribuição de construções e áreas verdes influenciam diretamente o comportamento térmico, o que está em conformidade com o resultado gerado à partir do modelo estudado. Ainda, conforme Da Costa, et. al, que realizou um estudo sobre as variações sazonais da ilha de calor urbana na cidade de Belém, Pará, a vegetação possui extrema importância na amenização de temperaturas máximas, o que contribui para a diminuição do desconforto humano.

\section{Conclusão}

Com a realização desse trabalho, foi perceptível a transferência de calor entre cidades (ilhas de calor) e 


\section{SEMANA DE ENGENHARIA QUÍMICA UFES}

\section{Blucher}

as florestas. Dados como a geração de calor de cada pessoa, fábrica e automóvel nas cidades foi um ponto apresentado durante $o$ trabalho que não é de conhecimento das maiorias das pessoas, que deveriam estar cientes, para que pudessem reduzir essas gerações a ponto de termos um ambiente com temperaturas mais amenas.

Pelo estudo dos resultados percebeu-se que as florestas tem grande poder de resfriamento, tornandose essenciais para que as temperaturas não atinjam valores ainda mais alarmantes e prejudiciais ao ser humano e a natureza.

\section{Referências}

[1] LEAL, Luciana. A influência da vegetação no clima urbano da cidade de Curitiba. 20/06/2012. 172. Tese Universidade Federal do Paraná.
[2] DA COSTA, Antonio C. L.; MATTOS, Arthur. Variações sazonais da ilha de calor urbana na cidade de Belém do Para, Universidade Federal do Pará.

[3] INCROPERA, Frank P.; DEWITT, David P.; BERGMAN, Theodore L.; LAVINE, Adrienne S. Fundamentos de transferência de calor e da massa. Tradução e revisão técnica: Eduardo Mach Queiroz, Fernando Luiz Pellegrini Pessoa. Rio de Janeiro: LTC, 2008.

[4] ALMEIDA, Domingos P. F.. Apontamentos de apoio às aulas de tecnologia pós-colheita. PSICOMETRIA, Universidade do Porto. 2004. 